\title{
Visualization of spatiotemporal data with different topology
}

\author{
Alena Vondrákováa ${ }^{\mathrm{a} *}$, Vít Pászto $^{\mathrm{a}}$ \\ ${ }^{a}$ Department of Geoinformatics, Palacký University Olomouc, Czechia, Alena Vondráková - alena.vondrakova@ upol.cz, Vít Pászto \\ - vit.paszto@upol.cz \\ * Corresponding author
}

Keywords: geodata, administrative boundaries, spatio-temporal analysis

\begin{abstract}
:
The availability of advanced technologies, the time of information society, and also the development of geographic information systems, have brought a lot of spatial data in most disciplines, which are carefully stored in recent decades and allow us spatiotemporal analysis and visualization. However, for long-term analyses and the synthesis of analysed data, including cartographic synthesis processes, it is a big problem when spatiotemporal data have different topologies at different times. And this does not only mean the correction of borders in the sense of clarification or other distinction. The problem is when, for example, the small administrative units is subject to significant temporal changes. Municipalities are divided and merged, while data are always stored for the topology of a particular year or period.

The contribution presents a way to solve such a situation on the example of the Czech Republic. Data from 25 years' period are adjusted so that it is possible to calculate and visualize long-term trends and analyses. In the case of the case study, these are spatial data of more than 6,000 municipalities, with changes in more than 200 cases during the observed period. In addition to the spatial component, there were also changes in identifiers, which are a common means of joining data. For example, when the name of the municipality was changed and the newly "created" municipality also received new identification code. All these problems are solved by the so-called "super layer", which represents aggregation to the smallest possible extent so that the analyses performed are carried out on the most detailed possible scale without missing data in partial periods.
\end{abstract}

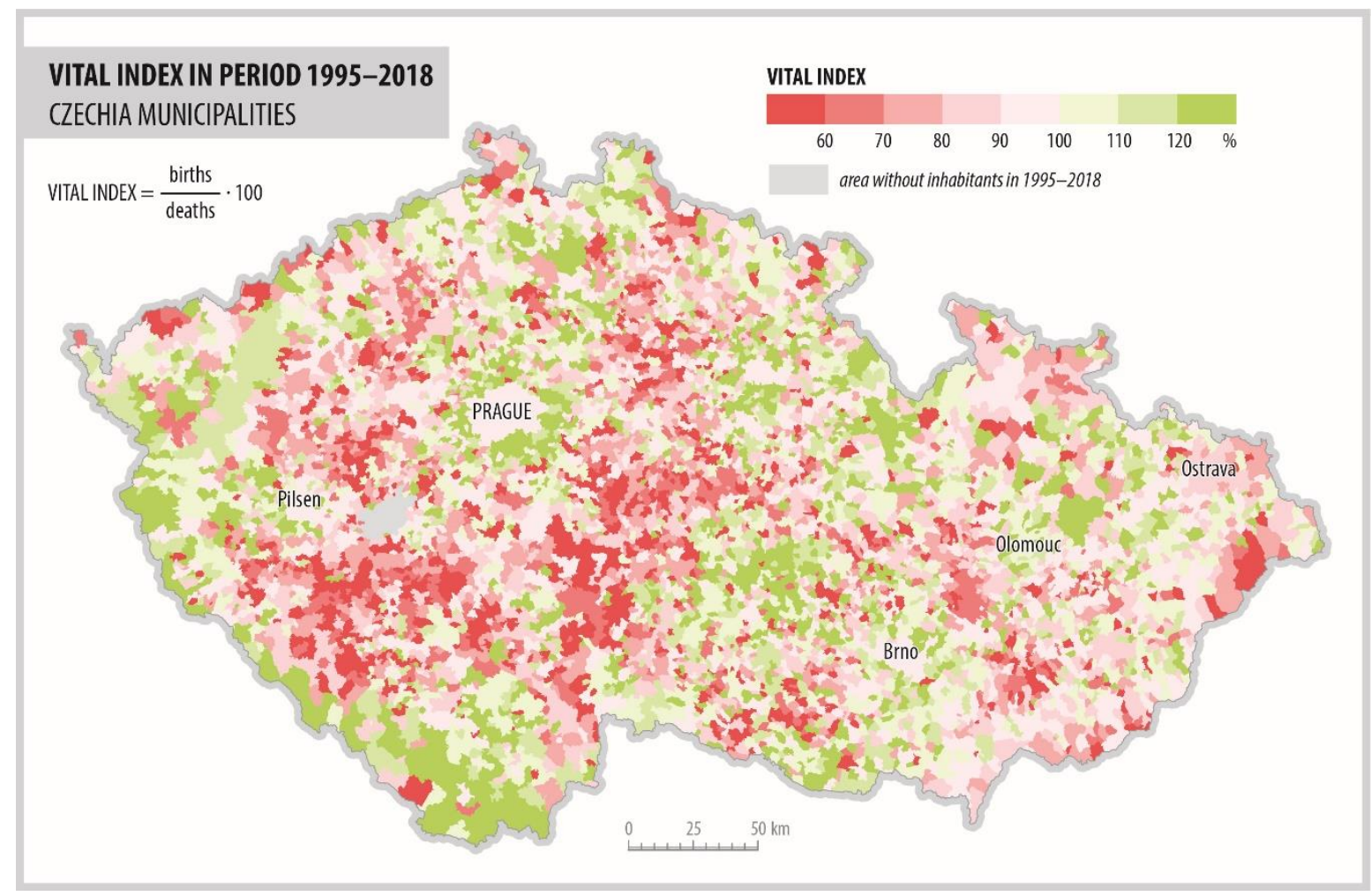

Figure 1. Map using "super-layer" for visualization geodata from 25 years period. 
The project goal is to explore various geodemographic processes at a very detailed level, specifically at NUTS (Nomenclature of Territorial Units for Statistics) local administrative units 2 (LAU2) commonly used in European Union for statistical purposes. Our presented dataset/approach unified municipal administrative units allowing analyses of data as they change over time. We used a principle of "common spatial denominator", i.e. we used data aggregation into larger units with stable boundary.

\section{Acknowledgements}

This contribution was supported by the project Nr. 18-12166S (Spatial differentiation and visualization of geodemographic processes with a focus on households in an ageing society in the Czech Republic), funded by The Czech Science Foundation. 